\section{Revista 2020 \\ Med Vol. 28(1)}

Enero-Junio 2020
-

ISSN: 0121-5256 - ISSN-e: 1909-7700

pp. 59 - 67

Editorial Neogranadina

\title{
Asociación VACTERL de presentación inusual: reporte de caso*
}

\author{
Natalia Andrea Torres Valencia - Myriam Pardo \\ - Javier Llanos ${ }^{c}$ - Leonardo Sierrad - Yenifer Alarcóne
}

Resumen: En el contexto de Asociación VACTERL, el diagnóstico prenatal de atresia esofágica concomitante con atresia duodenal es poco común. En el presente artículo se realiza el reporte de un caso con la descripción de los hallazgos ecográficos encontrados a partir semana doce de gestación, con la aparición del signo de doble burbuja intraabdominal compatible con atresia duodenal y una arteria umbilical única; y hacia la semana 31 el hallazgo de imagen anecóica y dilatación esofágica del tercio distal con comunicación con la cámara gástrica en corte longitudinal, representando una atresia esofágica, asociada además a polihidramnios. Desde el momento del nacimiento por examen físico y estudios complementarios, se evidenció además ano imperforado con fístula recto vestibular, arcos costales derechos fusionados y hemivértebras. Por los anteriores hallazgos clínicos y radiológicos, se considera que se trata de una asociación VACTERL; sin embargo, sin alteraciones cardiacas, que es una de las características más frecuentemente encontradas. Se realiza una revisión del estado del arte con respecto a la asociación VACTERL y el diagnóstico prenatal de la atresia esofágica y duodenal.

Palabras clave: Asociación VACTERL; diagnóstico prenatal; alteraciones congénitas

Fecha de recepción: 25/11/2018. Fecha de aprobación: 05/05/2020.

Disponible en línea: 11/09/2020

Cómo citar: Torres Valencia NA, Pardo M, Llanos J, Sierra L, Alarcón Y. Asociación VACTERL de presentación inusual: reporte de caso. Rev. Med. [Internet]. 11 de septiembre de 2020 [citado 11 de septiembre de 2020];28(1). Disponible en: https://revistas.unimilitar.edu.co/index.php/rmed/article/ view/3217

* $\quad$ Reporte de caso.

a Autor de correspondencia. MD, residente de Ginecología y Obstetricia.

Hospital Militar Central, Bogotá, Colombia. Correo electrónico: natas_055@hotmail.com

b MD, pediatra, perinatóloga. Hospital Militar Central, Bogotá, Colombia.

Correo electrónico: paquitaconnie@gmail.com

c MD, pediatra, perinatólogo. Hospital Militar Central, Bogotá, Colombia.

Correo electrónico: Ilanosbuelvas@gmail.com

d MD, ginecólogo, obstetra, ecografista. Hospital Militar Central, Bogotá, Colombia.

Correo electrónico: leonardosierra2002@hotmail.com

e MD, pediatra fellow en Neonatología. Hospital Militar Central, Bogotá, Colombia.

Correo electrónico: yefalarcon@gmail.com 


\title{
VACTERL Association with Unusual Presentation: Case Report
}

\begin{abstract}
In the context of the VACTERL Association, prenatal diagnosis of concomitant esophageal atresia with duodenal atresia is rare. In this article, a case report is described with the description of the ultrasound findings found from week twelve of gestation, with the appearance of the intra-abdominal double bubble sign compatible with duodenal atresia and a single umbilical artery; and towards week 31 the finding of anechoic image and esophageal dilation of the distal-third with communication with the gastric chamber in longitudinal section, representing esophageal atresia, also associated with polyhydramnios. Physical examination and complementary studies from birth showed an imperforate anus with a rectovestibular fistula, and hemivertebrae. Based on the above clinical and radiological findings, it is considered to be a VACTERL association; however, without cardiac abnormalities, which is one of the most frequently found characteristics. A review of the state of the art is carried out with regard to the VACTERL association and the prenatal diagnosis of esophageal and duodenal atresia.
\end{abstract}

Keywords: VACTERL association, prenatal diagnosis; congenital disorders

\section{AsSOciação VACTERL de apresentação rara: relato de caso}

Resumo: No contexto da Associação VACTERL, o diagnóstico pré-natal de atrésia do esôfago concomitante com atrésia de duodeno é pouco comum. Neste artigo, é realizado relato de um caso com a descrição dos achados ecográficos encontrados a partir da $12^{a}$ semana de gestação, com o surgimento do sinal de dupla bolha intra-abdominal compatível com a atrésia duodenal e uma artéria umbilical única; na $31^{\text {a }}$ semana, o achado de imagem anecoica e dilatação esofágica do rádio distal com comunicação com a câmara gástrica em corte longitudinal, o que representa atrésia esofágica, associada, ainda, a polidrâmnio. Desde o momento do nascimento por exame físico e estudos complementares, é evidenciado ânus imperfurado com fístula retal vestibular, arcos costais direitos fusionados e hemivértebras. A partir dos achados clínicos e radiológicos, é considerado que se trata de uma associação VACTERL; contudo, sem alterações cardíacas, que é uma das características mais frequentemente encontradas. É realizada uma revisão do estado da arte a respeito da associação VACTERL e o diagnóstico pré-natal da atrésia esofágica e duodenal.

Palavras-chave: Associação VACTERL; diagnóstico pré-natal; alterações congênitas 


\section{Introducción}

El diagnóstico prenatal es el conjunto de medidas clínicas y paraclínicas que permiten la identificación de alteraciones congénitas con el fin de determinar conductas a lo largo de la gestación y la preparación para la atención integral (1). Las malformaciones congénitas son infrecuentes y representan menos del $2 \%$ de los recién nacidos vivos (2).

Se describe como asociación la presencia de malformaciones congénitas en diferentes órganos y sistemas que pueden asociarse a otras sin que pueda existir un factor desencadenante común (1). Es así como la asociación VACTERL combina de manera no aleatoria múltiples malformaciones congénitas, que incluyen alteraciones vertebrales, anales, cardiacas, traqueoesofágicas, renales y de extremidades; no hay un consenso de criterios diagnósticos estrictos, pero la mayoría de clínicos e investigadores requieren la presencia de al menos tres de estas malformaciones para hacer el diagnóstico (1). La aparición conjunta con atresia duodenal ocurre solo en el $5 \%$ de los casos y es aún más infrecuente la identificación prenatal temprana de estas características $(2,3)$.

El propósito del presente reporte es brindar al lector los elementos de juicio que le permitan reconocer la asociación, especialmente cuando se evidencie la presencia concomitante de atresia esofágica y atresia duodenal, situación infrecuente en la descripción de la patología.

\section{Reporte de caso}

Una paciente de diecinueve años primigestante ingresa al servicio de urgencias de Ginecología y Obstetricia del Hospital Militar Central con un embarazo de 12 y $1 / 7$ semanas, con un cuadro clínico compatible con una amenaza de aborto; sin antecedentes personales o familiares de relevancia y sin exposición a ningún teratógeno conocido. Se solicita ecografía obstétrica en la que se reporta un feto con LCC de $58 \mathrm{~mm}$ y, como hallazgo incidental, la presencia del signo de doble burbuja intraabdominal, compatible con atresia duodenal. Se remite al paciente al servicio de Medicina Materno Fetal de la institución (Figura 1).
Posteriormente, en la ecografía de tamizaje 11-13,6, se reconoce nuevamente el signo de doble burbuja abdominal en un feto con LCC de $64 \mathrm{~mm}$, arteria umbilical única, con hueso nasal presente y una sonolucencia nucal de $1,1 \mathrm{~mm}$.

Se solicita amniocentesis diagnóstica y el cariotipo reporta $46 \mathrm{xx}$ sin aberraciones estructurales ni numéricas.

En la ecografía obstétrica de detalle anatómico realizada a las veintiún semanas de gestación, hay persistencia de los hallazgos ecográficos de doble burbuja intraabdominal, presencia de arteria umbilical única y un perfil de crecimiento normal (Figura 2).

En una ecografía de seguimiento realizada en la semana 31, además se logra evidenciar en el mediastino, una imagen anecoica no vascularizada al Doppler color, con relación a dilatación esofágica en el tercio distal, la cual se asoció a polihidramnios, con lo que se hizo una impresión diagnóstica de atresia esofágica asociada (Figuras 3 y 4 ).

Hacia la semana 33 de gestación, ingresa nuevamente la paciente al servicio de urgencias de Ginecología y Obstetricia, con amniorrea evidente $y$ en trabajo de parto en fase latente. Se realiza cesárea de urgencias por estado fetal no tranquilizador según reporte de monitoría fetal electrónica. Se recibe recién nacido femenino Ballard para 33 semanas, con peso de 1.780 g considerado adecuado para edad gestacional, con adaptación conducida y posterior falla ventilatoria, requiriendo intubación orotraqueal. En el examen físico se evidenció implantación baja de las orejas, arteria umbilical única, abdomen distendido, ano imperforado con fístula recto vestibular y genitales femeninos externos normoconfigurados. Es trasladada a unidad de cuidados intensivos neonatales, en donde se realiza radiografía de tórax y abdomen (Figura 5), la cual muestra arcos costales derechos fusionados, hemivértebras y ausencia de gas en cámara gástrica, considerando una atresia esofágica tipo 1. Dados los antecedentes prenatales, los hallazgos clínicos e imagenológicos, se considera una asociación VACTERL y se decide completar el estudio de posibles anormalidades asociadas, realizando ecocardiograma, 
ecografía transfontanelar, ecografía renal y vías urinarias, todas reportadas normal. Fue llevada a su primera cirugía a las 48 horas de vida, donde se le realizó gastrostomía, colostomía y resección de membrana duodenal. A los 11 días de vida se realizó inicio del estímulo enteral trófico por gastrostomía. En segundo tiempo quirúrgico, 34 días después, se realizó toracotomía derecha y anastomosis término terminal esofágica.

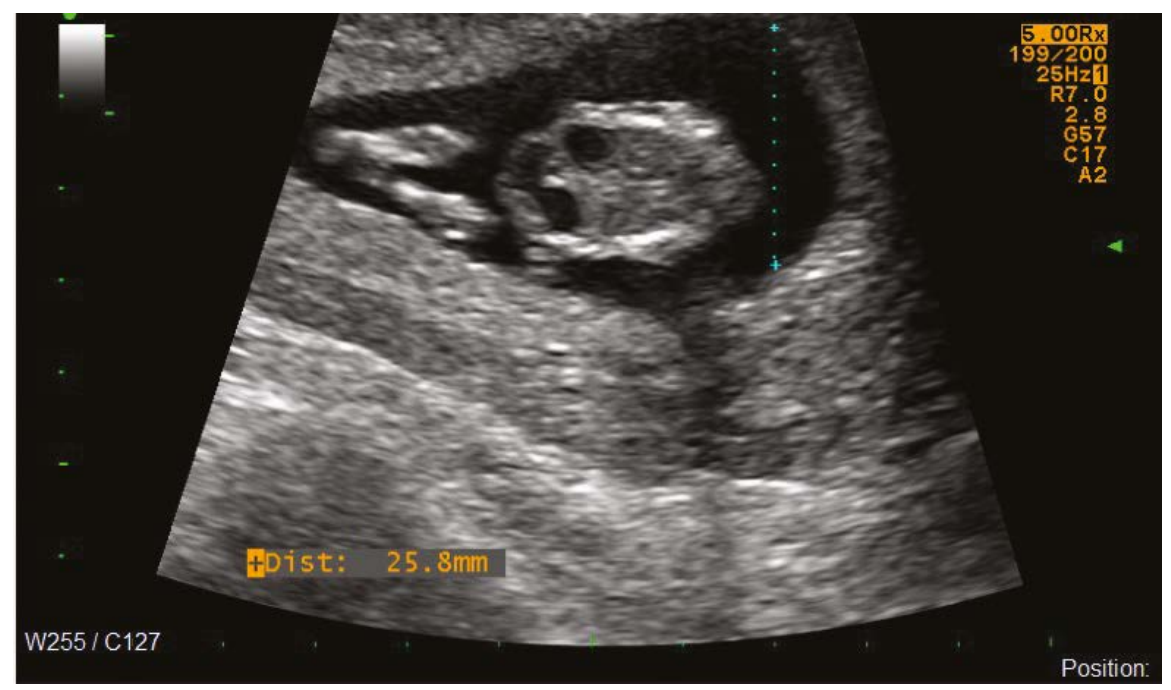

Figura 1. Embarazo de 12,1 semanas, signo de doble burbuja intraabdominal, compatible con atresia duodenal.

Fuente: Servicio de Urgencias Ginecología y Obstetricia, Hospital Militar Central Bogotá, Colombia.

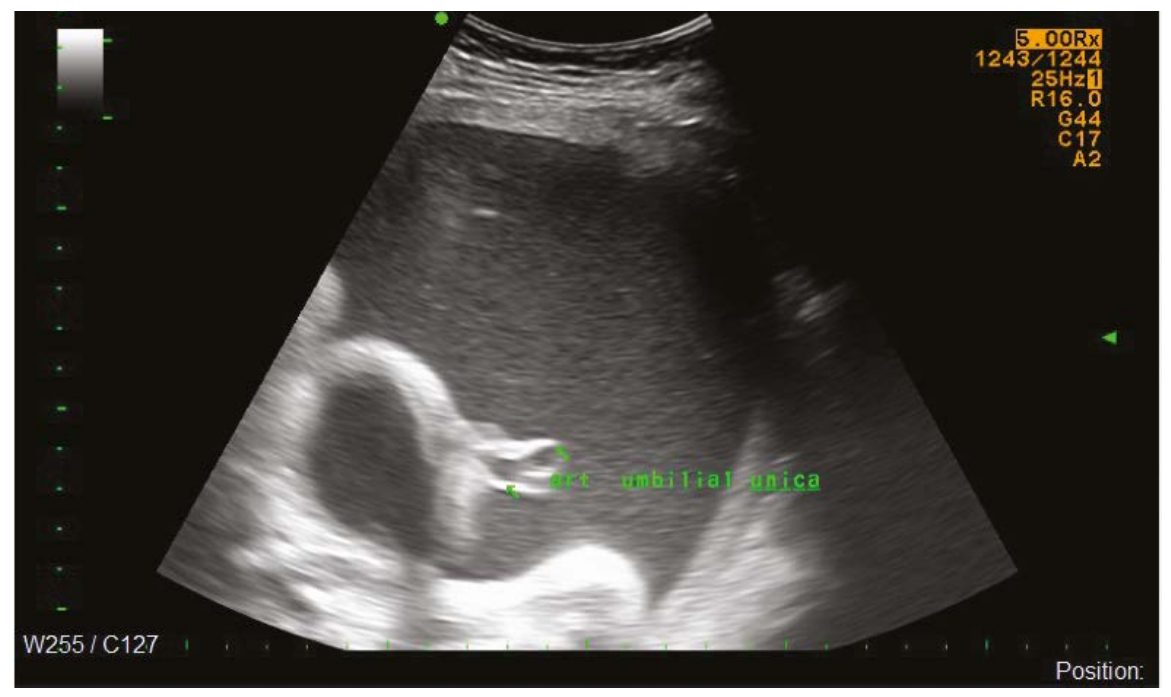

Figura 2. Embarazo de 31,5 semanas, arteria umbilical única.

Fuente: Servicio de Ecografía Ginecología y Obstetricia, Hospital Militar Central Bogotá,Colombia. 


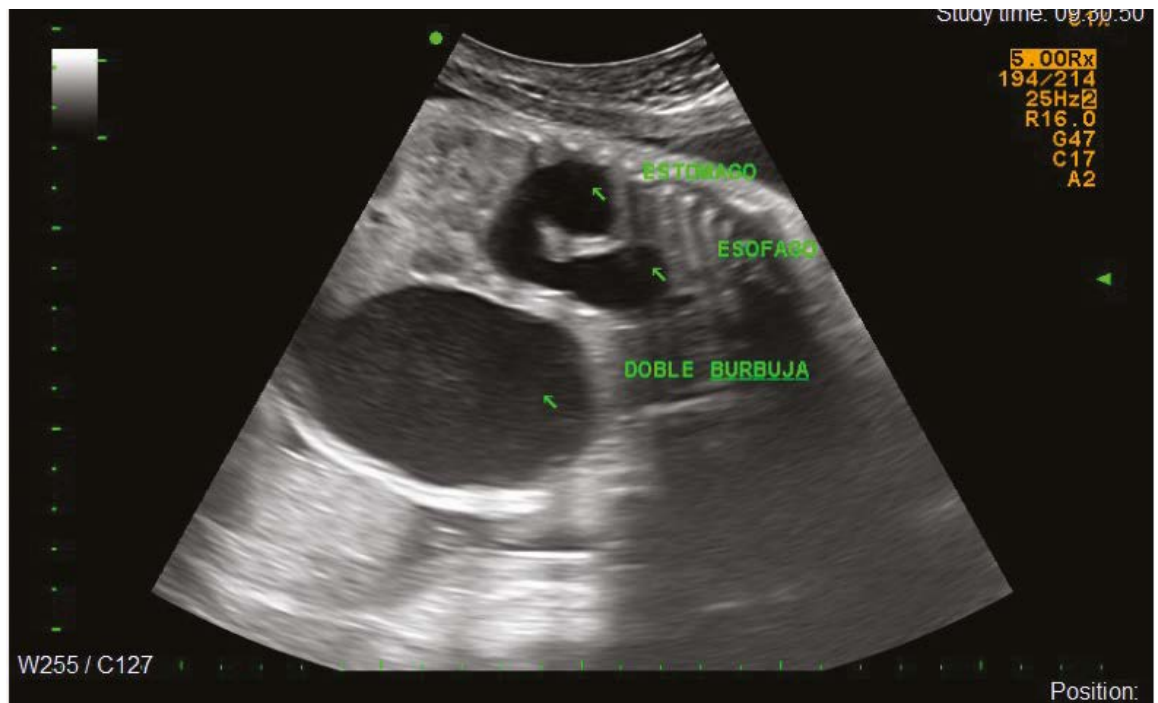

Figura 3. Embarazo de 31,5 semanas, signo de doble burbuja intraabdominal, dilatación esofágica en el tercio distal; corte longitudinal.

Fuente: Servicio de Ecografía Ginecología y Obstetricia, Hospital Militar Central Bogotá, Colombia.

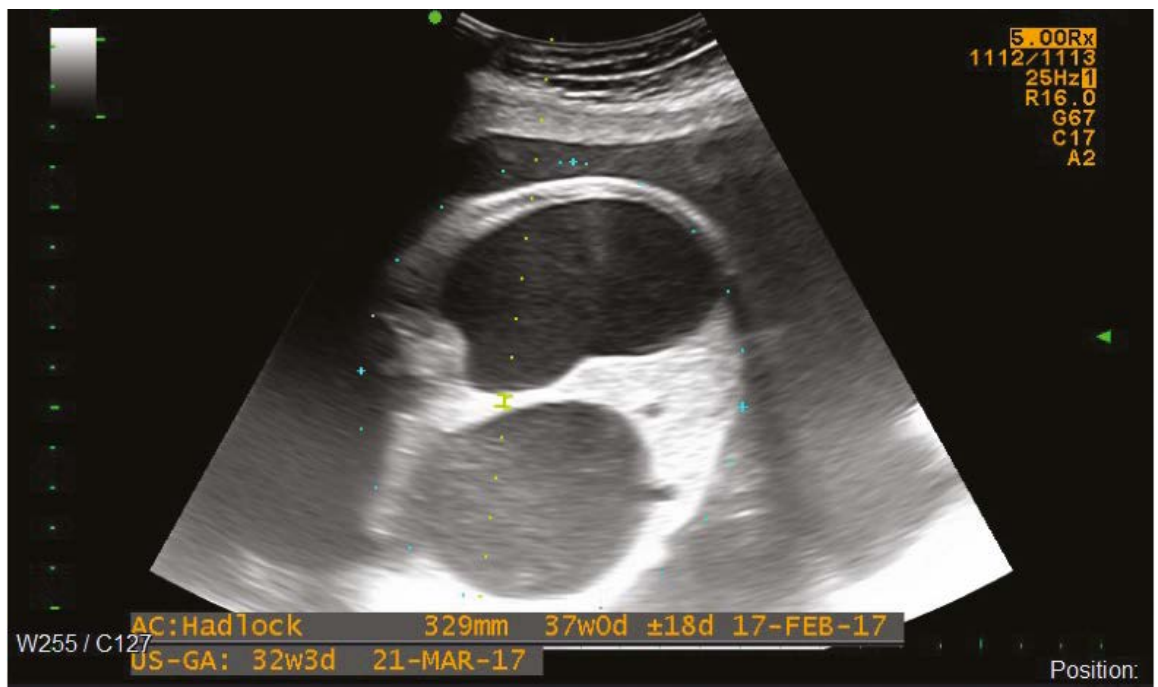

Figura 4. Embarazo de 31,5 semanas, signo de doble burbuja intraabdominal, corte transversal abdominal.

Fuente: Servicio de Ecografía Ginecología y Obstetricia, Hospital Militar Central Bogotá, Colombia. 


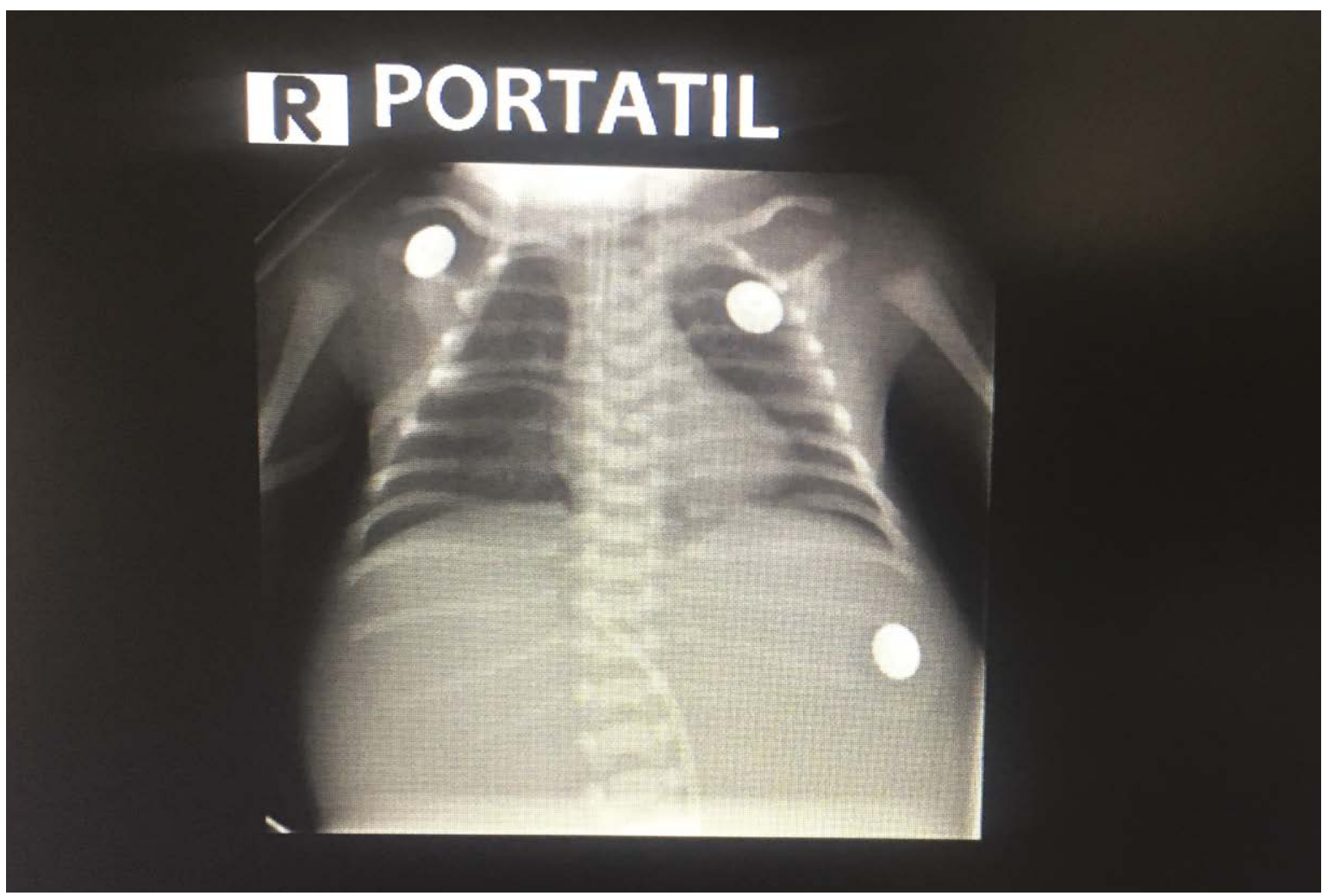

Figura 5. Rx de tórax del recién nacido: presencia de arcos costales derechos fusionados, hemivértebras y ausencia de gas en cámara gástrica considerando una atresia esofágica tipo 1.

Fuente: Hospital Militar Central Bogotá, Colombia.

\section{Discusión}

En 1973 Quan y Smith describieron por primera vez la asociación vaTER como una coocurrencia no aleatoria de cinco defectos: anomalías vertebrales (v), atresia anal (A), atresia esofágica (TE) y anormalidades radiales y renales (R) (4). Posteriormente en 1974, Temtamy y Miller incluyeron los defectos cardiacos (5), lo que cambió el acrónimo a VACTERL. Esta asociación tiene una incidencia estimada entre 1 / 10.000 y 1 / 40.000 nacidos vivos, pero solo el $1 \%$ de los casos tiene presente todas las anomalías descritas (6). Es una condición más común en varones que mujeres (7).

Dado el amplio espectro de los defectos, se sugiere una ocurrencia defectuosa del desarrollo durante la embriogénesis temprana, que afecta las estructuras derivadas del mesodermo embrionario (6).

La mayoría de los casos son esporádicos, pero se han descrito influencias ambientales como la diabetes pregestacional y la exposición intrauterina a estrógenos/progesterona, estatinas y doxorrubicina $(1,6,8)$.

En la mayoría de los estudios, las anormalidades vertebrales, que usualmente se acompañan de anormalidades en las costillas, se han reportado en el 60-80\% de los pacientes afectados por la asociación VACTERL; estas usualmente incluyen defectos de fragmentación como hemivértebra, fusiones vertebrales, vértebra supernumeraria o ausente y otras formas de displasia vertebral $(6,7)$.

Una anotación especial se debe hacer con respecto a la presencia de arteria umbilical única, la cual es un hallazgo frecuente en esta asociación y que desde los últimos años hace parte de la definición dentro de la letra $\mathrm{v}$ del acrónimo para anomalías vertebrales o vasculares (7).

Con respecto a las malformaciones cardiacas, estas son reportadas en el 40-80\% de estos pacientes; aunque algunos autores sugieren no 
considerarla en los criterios diagnósticos, ya que las anomalías estructurales del corazón no son más comunes en la asociación VACTERL que en otros desordenes con múltiples malformaciones. Estas anormalidades abarcan desde defectos estructurales severos incompatibles con la vida, hasta defectos anatómicos sutiles que se reconocen en la vida adulta $(9,10)$; las más frecuentes corresponden a los defectos septales ventriculares, defectos atriales y la tetralogía de Fallot $(2,13)$. Siendo las malformaciones cardiacas una de las que se reportan con mayor frecuencia en la asociación VACTERL, nuestro caso no cuenta con ellas y esto tiene gran relevancia en el pronóstico del paciente.

Las anormalidades renales pueden incluir la agenesia renal unilateral o bilateral en los casos severos, riñón en herradura o riñones quísticos. Estas son reportadas en el 50-80 \% de los casos de la asociación VACTERL $(7,10)$.

Las malformaciones de las extremidades han sido reportadas en 40-50 \% de estos pacientes. Clásicamente fueron definidas como malformaciones radiales, pero incluyen también aplasia o hipoplasia de los pulgares, polidactilia y alteraciones de las extremidades inferiores (9).

$\mathrm{La}$ atresia esofágica es en realidad un espectro reconocido de deformidades, con una variedad de combinaciones que involucran la atresia esofágica y la fístula traqueoesofágica; de estas, la variante más común, en el $85 \%$ de los casos, es la combinación de atresia esofágica proximal con fístula traqueoesofágica distal $(7,9)$. La capacidad para solucionar esta malformación, la supervivencia de estos neonatos y la calidad de vida futura, son indicadores de la eficacia de la atención hospitalaria. Para el diagnóstico prenatal, en el ultrasonido obstétrico es frecuente el hallazgo de polihidramnios y ausencia de la burbuja gástrica, signos que podrían verse en los fetos desde la semana 15 de gestación, con un VPP de 40-60\% en la mayoría de las series $(7,11)$. Se puede ver en algunos casos el signo de la bolsa ciega esofágica a nivel cervical o en el mediastino, lo que aumenta la precisión diagnóstica a un 95-100 \% (7,11). Esta anomalía aparece en la asociación VACTERL con una frecuencia de 50 a $80 \%$ de los casos, tratándose de la relación más ampliamente reconocida entre atresia esofági$\mathrm{ca} /$ fístula traqueoesofágica y otras anormalidades congénitas $(11,12)$.

El ano imperforado ocurre en el 55 a $90 \%$ de los casos afectados por la asociación. La imperforación completa del ano generalmente se descubre en el periodo posnatal inmediato (13). Asociadas a las alteraciones anorrectales, puede haber anomalías genitourinarias con una frecuencia del $25 \%$ y con un amplio rango de defectos posibles, desde una fístula del tracto genitourinario al tracto anorrectal, hasta genitales ambiguos, criptorquidia o hipospadias (10).

Existen otras anomalías gastrointestinales (diferentes a los defectos anorrectales) que se asocian a la presencia de atresia gastroesofágica/fístula traqueoesofágica, como lo es la atresia duodenal, la cual, aunque no se encuentra incluida dentro de la definición de la asociación VACTERL, se ha reportado hasta en el $5 \%$ de los casos $(2,7)$. Dicha anomalía tiene incidencia de 1 en 2.500 a 5.000 nacidos vivos. Ocurre usualmente en la segunda porción del duodeno. El diagnóstico ecográfico prenatal ha sido reportado en el $87 \%$ de los fetos afectados, en su mayoría por la detección del signo de doble burbuja intraabdominal; también se pueden evidenciar polihidramnios en el $40 \%$ de los casos a partir del segundo trimestre. Los signos ecográficos son relativamente lentos en desarrollarse, pero el signo de doble burbuja abdominal puede observarse tan pronto como a las 12 semanas de gestación $(10,14)$.

En una serie de 114 casos reportados por Ladd y Swenson se describe la incidencia de la asociación entre atresia esofágica y atresia duodenal en aproximadamente $5 \%$ de los casos de la asociación VACTERL, y es aún más baja la incidencia asociando además el ano imperforado, como lo es en el caso presentado (12). Teóricamente el diagnóstico de la atresia esofágica con fístula traqueoesofágica, atresia duodenal y malformación anorrectal puede realizarse de manera prenatal, con confirmación con estudio radiológico de tórax y abdomen en el neonato; sin embargo, hay gran dificultad especialmente cuando se trata de una atresia esofágica pura (15); en nuestro caso se reporta una atresia esofágica tipo 1. Identificamos varios casos en la literatura que se realizan en diagnóstico prenatal 
de atresia esofágica tipo 1 con atresia duodenal (1619); no obstante, solo en uno, así como en nuestro caso, se logran evidenciar todos los hallazgos ecográficos típicos, que incluyen polihidramnios, dilatación esofágica distal y signo de la doble burbuja intraabdominal (17).

El manejo de los pacientes con asociación VACTERL típicamente se centra en la corrección quirúrgica de las anomalías congénitas específicas en el periodo posnatal inmediato, seguido por el manejo médico a largo plazo de las secuelas de las malformaciones congénitas. Finalmente, es de importancia mencionar que esta asociación típicamente no tiene compromiso neurocognitivo. $(20,21)$.

\section{Referencias}

[1] Santos J, Nogueira R, Pinto R, Cerveira I, Pereira S. First trimester diagnosis of VACTERL Association [Internet]. Clinics and Practice. 2013;3(1):e5. DoI: https:// doi.org/10.4081/cp.2013.e5

[2] Choudhry MS, Rahman N, Boyd P, Lakhoo K. Duodenal atresia: associated anomalies, prenatal diagnosis and outcome [Internet]. Pediatr Surg Int. 2009;25(8):727-730. DOI: https://doi.org/10.1007/ s00383-009-2406-y

[3] Fujishiro E, Suzuki Y, Sato T, Kondo S, Miyachi M, Suzumori K. Characteristic findings for diagnosis of baby complicated with both VACTERL association and duodenal atresia [Internet]. Fetal Diagn Ther. 2004;19(2):134-137. DoI: https://doi. org/10.1159/000075137

[4] Quan L, Smith DW. The VATER association: vertebral defects, anal atresia, tracheoesophageal fistula with esophageal atresia, radial dysplasia [Internet]. Birth Defects. 1972;8:75-78. DoI: https://doi.org/10.1016/ s0022-3476(73)80024-1

[5] Temtamy SA, Miller JD. Extending the scope of vATER association: Definition of the VATER association: Definition of the VATER syndrome [Internet]. J Pediatr. 1974;85(3):345-349. DoI: https://doi.org/10.1016/ S0022-3476(74)80113-7

[6] Solomon B, Raam MS, Pineda-Alvarez DE. Analysis of genitourinary anomalies in patients with VACTERL association [Internet]. Congenit Anom. 2011;51(2):87-91. DoI: https://doi.org/10.1111/j.1741-4520.2010.00303.x
[7] Martin R, Fanaroff A, Walsh M. Neonatal-Perinatal Medicine. Amsterdam: Elsevier; 2020. 2008 p.

[8] Van de Putte R, van Rooij I, Roeleveld N, Loane M, Barisic I, Bergman J. de Walle $H$. Maternal diabetes and assisted reproductive techniques as maternal risk factors for the VACTERL association using data of EUROCAT registries and the distribution of VACTERL anomalies among VACTERL patients of EUROCAT [Internet]. European Journal of Medical Genetics. 2018;61(9):566-567. DoI: https://doi.org/10.1016/j.ejmg.2018.06.054

[9] Norton M. Callen's Ultrasonography in Obstetrics and Gynecology. Amsterdam: Elsevier; 2018. 1272 p.

[10] Copel J. Obstetric Imaging: Fetal Diagnosis and Care. Amsterdam: Elsevier; 2018. 848 p.

[11] Adrian S, Rami M, Yaron Z. Prenatal Identification of esophageal atresia: the role of ultrasonography for evaluation of functional anatomy[Internet]. Prenatal Diagnosis. 2002;22(8):669-674. DoI: https://doi. org/10.1002/pd.375

[12]Ladd WE, Swenson O. Esophageal atresia and tracheo-esophageal fistula [Internet]. Ann Surg. 1947;125(1):23-40. DoI: https://doi. org/10.1097/00000658-194701000-00002

[13] Lam YH, SHek T, Tang MH. Sonographic features of anal atresia at 12 weeks [Internet]. Ultrasound Obstet Gynecol. 2002;19(5):523-524. Dor: https://doi.org/10.1046/j.1469-0705.2002.00694.x

[14] Dundas KC, Walker J, Laing IA. Oesophageal and duodenal atresia suspected at the 12 week booking scan [Internet]. Br J Obsstet Gynecol. 2001;108(2):225-226. DOI: https://doi.org/10.1016/S0306-5456(00)00025-5

[15] Panda SS, Srinivas M, Bajpai M, Sharma N, Singh A, Baidya DK, et al. Esophageal atresia, duodenal atresia, and imperforate anus: Triple atresia [Internet]. J Clin Neonatol. 2015; 4(3):188-192. DoI: https://doi. org/10.4103/2249-4847.159907

[16] Duenhoelter JH, Santos-Ramos R, Rosenfeld CR, et al. Prenatal diagnosis of gastrointestinal tract obstruction [Internet]. Obstet Gynecol. 1976;47(5):618-620.

[17] Hayden CK Jr, Schwartz MZ, Davis M, et al. Combined esophageal and duodenal atresia: sonographic findings [Internet]. AJR Am J Roentgenol. 1983;140(2):225-226. DOI: https://doi.org/10.2214/ajr.140.2.225

[18] Estroff JA, Parad RB, Share JC, et al. Second trimester prenatal findings in duodenal and esophageal atresia without tracheoesophageal fistula [Internet]. J Ultrasound Med. 1994;13(5):375-379. DoI: https://d oi.org/10.7863/jum.1994.13.5.375 
[19] Marquette GP, Skoll MA, Yong SL, et al. First-trimester imaging of combined esophageal and duodenal atresia without a tracheoesophageal fistula [Internet]. J Ultrasound Med. 2005; 23(9):1232. DoI: https://doi. org/10.7863/jum.2004.23.9.1232

[20]Norton ME, Kuller JA, Dugoff L. Perinatal Genetics. Elsevier. 2020. 105-125.

[21] Anne DL, Carole G, Helene L. Prenatal diagnosis of the VACTERL association using routine ultrasound examination [Internet]. Clinical and Molecular Teratology. 2015;103(10):880-886. Dor: https://doi.org/10.1002/ bdra. 23346 